\title{
Plaque erosion is a major substrate for coronary thrombosis in acute myocardial infarction
}

\author{
E Arbustini, B Dal Bello, P Morbini, A P Burke, M Bocciarelli, G Specchia, R Virmani
}

\begin{abstract}
Objective-To evaluate the prevalence of plaque erosion as a substrate for coronary thrombosis.

Design-Pathological study in patients with acute myocardial infarction not treated with thrombolysis or coronary interventional procedures.

Patients-298 consecutive patients (189 men, mean (SD) age 66 (11) years; 109 women, 74 (8) years) dying in hospital between 1984 and 1996 from acute myocardial infarction, diagnosed by ECG changes and rise in cardiac enzymes.

Main outcome measures-Histopathological determination of plaque erosion as substrate for acute thrombosis; location and histological type of coronary thrombosis; acute and healed myocardial infarcts; ventricular rupture.

Results-Acute coronary thrombi were found in 291 hearts $(98 \%)$; in 74 cases $(25 \% ; 40 / 107$ women $(37.4 \%)$ and $34 / 184$ men $(18.5 \%) ; p=0.0004)$, the plaque substrate for thrombosis was erosion. Healed infarcts were found in $37.5 \%$ of men $v 22 \%$ of women $(p=0.01)$. Heart rupture was more common in women than in men $(22 \% v 10.5 \%, p=0.01)$. The distribution of infarcts, thrombus location, heart rupture, and healed infarcts was similar in cases of plaque rupture and plaque erosion.

Conclusions-Plaque erosion is an important substrate for coronary thrombosis in patients dying of acute myocardial infarction. Its prevalence is significantly higher in women than in men.

(Heart 1999;82:269-272)
\end{abstract}

Keywords: plaque erosion; rupture; acute myocardial infarction

Department of

Cardiology,

IRCCS-Policlinico San

Matteo

G Specchia

Department of

Cardiovascular

Pathology, Armed

Forces Institute of

Pathology,

Washington, DC, USA

A P Burke

R Virmani

Correspondence to:

Dr Arbustini.

email: e.arbustini@

smatteo.pv.it

Accepted for publication 10 June 1999

Plaque rupture is the major local substrate for acute coronary thrombosis, causing unstable angina, acute myocardial infarction, and sudden ischaemic death. ${ }^{1-3}$ The vulnerable plaque that is prone to rupture consists of a necrotic core with a thin fibrous cap infiltrated by inflammatory cells, ${ }^{4}$ especially metalloproteinase-rich macrophages. ${ }^{5}{ }^{6}$ The most frequent site of plaque rupture has been localised to the shoulder region, ${ }^{7}$ where thrombogenic, atheromatous material is exposed to luminal flow. ${ }^{8}$ The corresponding angiographic pattern is that of multiple luminal irregularities. ${ }^{9}$ Plaques that do not have a core or have a thick fibrous cap are not vulnerable to rupture.
It has recently been shown that acute coronary thrombosis causing sudden ischaemic death may arise from two different underlying plaque morphologies: superficial erosion and plaque rupture. ${ }^{1-3}$ Of 50 cases of coronary thrombi identified in subjects who died suddenly (mean (SD) age, 44 (7) years), $44 \%$ had plaque erosion and $56 \%$ ruptured plaques. ${ }^{10}$ This observation suggests a new pathogenic hypothesis for local thrombogenic substrates and widens the morphological spectrum of risky coronary lesions in vivo.

Our aim in the present study was to evaluate the prevalence of plaque erosion as a substrate for acute thrombosis in patients who died in the hospital from acute myocardial infarction and did not receive thrombolysis or any coronary interventional procedures.

\section{Methods}

PATHOLOGICAL SERIES

Since 1985, the entire epicardial coronary artery tree and necropsy heart samples from patients with and without a clinical diagnosis of ischaemic heart disease have been subjected to histopathological investigation in our institution. $^{11}$ Of 2304 consecutive hearts (1985-1996), 1335 were from patients with a clinical diagnosis of heart disease. We excluded all patients who died from non-cardiac causes with major non-cardiac pathology (cancer, pulmonary embolism, hepatic cirrhosis, cerebral haemorrhage, pancreatitis, and so on). Of the 1335 hearts, 685 were from patients with acute myocardial infarction.

To investigate the characteristics of plaque morphology in acute coronary thrombosis, we included patients who died from both $\mathrm{Q}$ wave and non- $Q$ wave acute myocardial infarction. Patients who had received thrombolytic treatment or myocardial revascularisation - surgical or percutaneous - within one month of the clinical diagnosis were excluded. We also excluded all patients who had had one or more
Table 1 Necropsy cases/year and cases of acute myocardial infarction suitable for study

\begin{tabular}{llll} 
Year & $\begin{array}{l}\text { Total necropsies } \\
(n)\end{array}$ & $\begin{array}{l}\text { Patients dying of } \\
\text { cardiac causes }\end{array}$ & $\begin{array}{l}\text { AMI patients } \\
\text { for study }\end{array}$ \\
\hline 1985 & 227 & 114 & 37 \\
1986 & 172 & 102 & 25 \\
1987 & 234 & 132 & 41 \\
1988 & 190 & 113 & 27 \\
1989 & 237 & 151 & 30 \\
1990 & 218 & 120 & 28 \\
1991 & 197 & 111 & 26 \\
1992 & 175 & 103 & 21 \\
1993 & 191 & 123 & 25 \\
1994 & 154 & 109 & 18 \\
1995 & 182 & 98 & 13 \\
1996 & 127 & 59 & 7
\end{tabular}

AMI, acute myocardial infarction. 

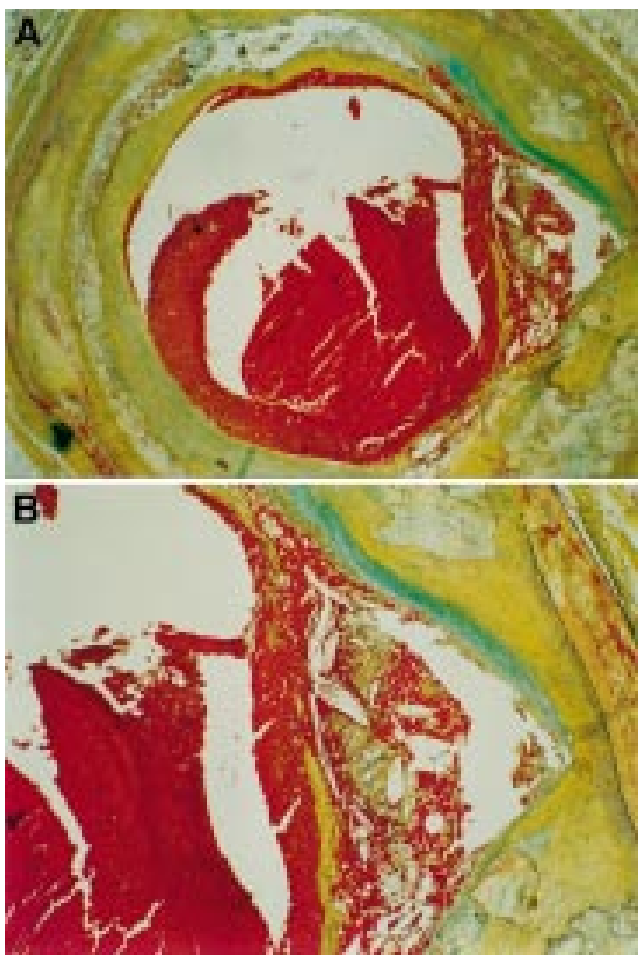

Figure 1 Typical plaque ulceration with haemorrhagic core, cap rupture, and luminal thrombus. (Movat Pentachrome stain; $A, \times 121 ; B, \times 80$.)

than one previous surgical revascularisation procedure and who had been on chronic pharmacological treatment with antiplatelet drugs. Furthermore, to achieve homogeneous sampling of the coronary trees, only hearts studied by a single investigator were included. Only 298 of the 685 cases met these inclusion criteria. There were 189 men $(63 \%$; mean (SD) age 66 (11) years) and 109 women (37\%; mean age 77 (8) years). The distribution of the cases per year is given in table 1 ; the number of cases decreased progressively between 1985 and 1996.

PATHOLOGICAL STUDY

The hearts were fixed in $10 \%$ buffered formalin overnight and the major epicardial coronary arteries were dissected free from the hearts according to previously described methods. ${ }^{311}$ The left main, left anterior descending, left circumflex, and right coronary arteries were excised intact, and the latter three arteries were divided into proximal, middle, and distal portions. In all cases, the first diagonal, first obtuse, and posterior descending branches were also excised and divided into 3-5 mm segments. When necessary, coronary arteries were decalcified before cutting, by previously reported methods. ${ }^{31}$ The major coronary arteries were sectioned at $5 \mathrm{~mm}$ intervals, embedded in paraffin, cut at $5 \mu \mathrm{m}$ thickness, and stained with haematoxylin-eosin and Movat Pentachrome. The ventricles were "bread loafed" at 1.5 to $2 \mathrm{~cm}$ intervals. Full thickness samples extending from endocardium to epicardium of the walls of the right and left ventricles were cut from each heart for histological study.

Coronary thrombi were identified by site and number in the coronary tree, and classified histologically as acute or recanalised/organised. Old recanalised thrombi were not considered further. The distribution of acute myocardial infarction and ventricular scars was correlated with the site of thrombosis, and the presence of ventricular wall rupture was determined pathologically. Coronary sections containing thrombi were identified by light microscopy and when evidence of rupture was not found in this first set of sections, serial cuts were done from the corresponding paraffin block to
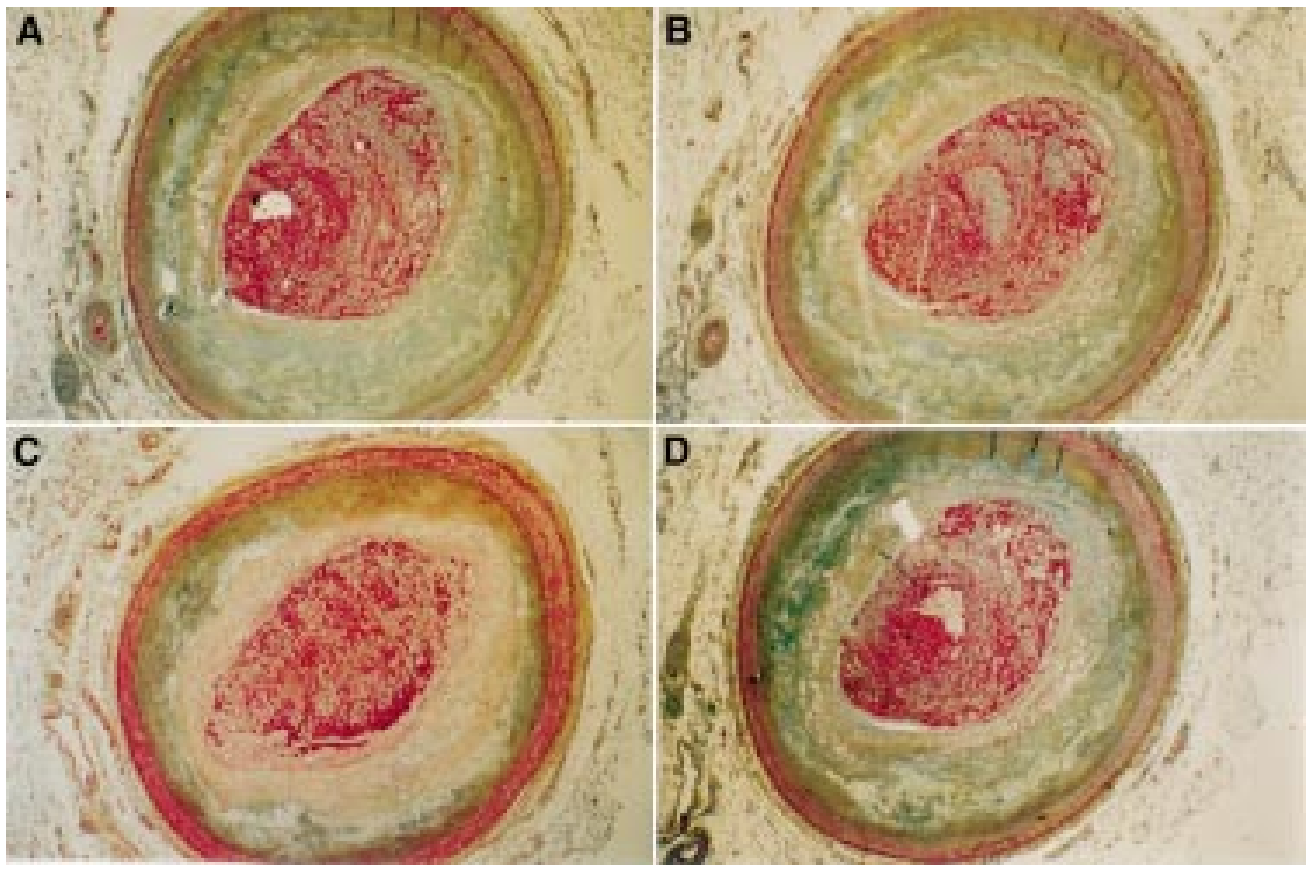

Figure 2 Plaque erosion: in multiple sections, four in the present figure, the thrombus outlines the profile of the plaque and there is no evidence of continuity between thrombus and plaque core. (Movat Pentachrome stain, $\times 84$.) 
Table 2 Erosion and rupture in plaques with acute thrombosis: distribution of patients, clinical data, and pathological findings

\begin{tabular}{lccl}
\hline & $\begin{array}{l}\text { Plaque erosion } \\
(n=74)\end{array}$ & $\begin{array}{c}\text { Plaque rupture } \\
(n=217)\end{array}$ & $p$ Value \\
\hline $\begin{array}{l}\text { Male/female } \\
\text { Age (mean (SD)) (years) }\end{array}$ & $34 / 40$ & $150 / 67$ & 0.0004 \\
AMI site & $70(9)$ & $68(11)$ & NS \\
$\quad$ Anterolateral & $49(66.2)$ & $119(54.8)$ & NS \\
$\quad$ Lateral & $3(4.1)$ & $22(10.1)$ & NS \\
$\quad$ Posterior & $22(29.7)$ & $76(35.1)$ & NS \\
Heart rupture & $17(22.9)$ & $27(12.4)$ & NS \\
Non-Q wave AMI (thrombi 11/15) & $1(1.3)$ & $10(4.6)$ & NS \\
Scars & $21(28.4)$ & $74(34.1)$ & NS \\
Multiple thrombi & $11(14.9)$ & $18(8.3)$ & NS \\
\hline
\end{tabular}

Values are $\mathrm{n}(\%)$ except where stated.

AMI, acute myocardial infarction.

examine the entire segment of coronary artery containing the thrombus.

DEFINITION OF PLAQUE RUPTURE AND EROSION

Ruptures were defined as the presence of a luminal thrombus in continuity with the necrotic core and an interrupted plaque cap ${ }^{10}$ (fig 1). When necrotic material was mixed with thrombotic material at any level of the thrombus, the thrombus was considered to have been the result of plaque rupture. A thrombus was assumed to have been the result of plaque erosion when there was no continuity between the thrombus and the necrotic core and where the thrombus was in direct contact with the fibrointimal plaque (fig 2). ${ }^{10}$

A few plaques which had indirect signs of ulceration exclusively, without evidence of contiguity between atheromatous and thrombotic material or cap interruption, were assigned to the plaque rupture group.

\section{STATISTICAL ANALYSIS}

Numerical data are presented as mean (SD). Continuous variables were compared with analysis of variance, and categorical variables were compared by the $\chi^{2}$ test (Statview Software, Version 4.01). A probability (p) value of $\leqslant 0.05$ was considered significant.

\section{Results}

Acute coronary thrombi were identified in 291 coronary arteries. In 74 plaques, the lesion underlying the thrombus was defined as erosion, based on the above criteria. In these plaques we did not find any direct or indirect evidence of continuity between atheromatous and thrombotic material (fig 2). Thus overall, $25 \%$ of the thrombi found in patients with acute myocardial infarction occurred over non-ruptured plaques (plaque erosion), while $75 \%$ occurred over ruptured plaques.

Plaque erosion was more common in women: 40 of the 107 women with coronary thrombosis $(37.4 \%)$ and 34 of the 184 men $(18.5 \%)$ showed features of erosion $(p=0.0004)$. Sex was the only variable significantly associated with plaque erosion; infarct size, more than one thrombus in the coronary

Table 3 Myocardial scar and heart rupture frequency in the overall population

\begin{tabular}{llll}
\hline & Men & Women & p Value \\
\hline Myocardial scars & $71 / 189(37.5 \%)$ & $24 / 109(22 \%)$ & 0.01 \\
Heart rupture & $20 / 189(10.5 \%)$ & $24 / 109(22 \%)$ & 0.01 \\
\hline
\end{tabular}

tree, and the specific artery containing thrombus were distributed similarly among the two types of plaque lesion (table 2).

Heart rupture was more common in women $(22 \% ; 24$ of 109$)$ than in men $(10.5 \% ; 20$ of 189) $(\mathrm{p}=0.01)$ (table 3$)$. Rupture involved the free ventricular wall in 38 patients, the interventricular septum in five, and the papillary muscle in one. The site of rupture was not significantly associated with the type of plaque lesion underlying the thrombus.

Myocardial scars were more common in men $(37.5 \% ; 71$ of 189$)$ than in women $(22 \% ; 24$ of 109) $(\mathrm{p}=0.01)($ table 3$)$. No association was found between erosion/rupture and presence of previous scars.

Multiple thrombi were found in 29 coronary trees (17 from men and 12 from women). In three of the 29 patients, all men, we found three thrombi in three locations in the coronary tree. No significant association was found between erosion/rupture and multiple thrombi in the coronary arteries.

\section{Discussion}

We have documented that a relatively large proportion $(25 \%)$ of plaques underlying coronary thrombosis in patients with acute myocardial infarction do not show any rupture on multiple serial sectioning. Plaque erosion could only be identified by histopathological criteria. This current study of patients who died in hospital from clinically documented acute myocardial infarction confirms previous observations in sudden coronary death victims, in whom plaque erosion was found to underlie acute coronary thrombosis in about $44 \%$ of cases. ${ }^{10}$ The implications of these findings are of practical importance. Plaque erosion may occur in fibrous plaques in which the atheromatous component is absent, or in plaques in which the core is separated from the lumen by a thick fibrous cap. The profile of a vulnerable plaque is of a large eccentric lesion with a sizeable atheromatous core, a thin cap, a weak shoulder, and inflammatory cells clustered within the shoulder. However, even plaques without a core, or with a thick cap, may form the substrate for an acute occlusive coronary event. Therefore, coronary lesions with smooth morphology on angiography may have thrombogenic potential, and the predictive value of angiographic characterisation of vulnerable lesions only applies to plaques in which the pattern is one of plaque rupture. ${ }^{19}$ Furthermore, one cannot rely on multiple irregularities as a pattern common to all thrombi in patients with acute myocardial infarction in predicting rapid, non-linear plaque growth. ${ }^{12}{ }^{13}$

We have identified plaque erosion in one quarter of all the plaques underlying acute thrombus in patients with acute myocardial infarction. In our series, however, this proportion probably underestimates the true prevalence of this pattern because all "uncertain" lesions were classed as plaque ruptures. Furthermore, plaques with some evidence of haemorrhage but without identification of the point of rupture were also classed as ruptured plaques. In a plaque with luminal thrombus, if 
the atheromatous core showed haemorrhage our interpretation was that the point of rupture had been missed.

The prevalence of plaque erosion in women was significantly higher than in men $(37.4 \% v$ $18.5 \%, \mathrm{p}=0.0001)$. These data agree with previous observations by Farb et $a l,{ }^{10}$ who found a much higher prevalence of erosion in women who died suddenly than reported in men $(p=0.001)$. This observation suggests that in women, plaques are morphologically different from those observed in men or, alternatively, that even within similar lesions the thrombogenic potential is greater in affected women than in men. This observation merits further investigation both for differences in local plaque substrate and in relation to the mechanism of thrombosis. Recent studies have shown that the risk of erosion is greater in female smokers less than 50 years old than in older women. ${ }^{14}$ These observations suggest that both the risk factors for the disease and the disease itself are, at least in some respects, different in the two sexes.

In the current study, the prevalence of cardiac rupture was significantly higher in women than in men. There is little controversy that cardiac rupture affects women at least twice as often as men. ${ }^{15}$ On the other hand, our study corroborates the observation that myocardial scars, either subendocardial or transmural, are more common in men than in women. ${ }^{16}$ This may signify a "longer" disease history in the male population, with a higher incidence of adverse events. It has been reported that cardiac rupture occurs more often in hearts in which acute myocardial infarction is the first clinical ischaemic event. ${ }^{17}$ In other words, an acute myocardial infarct undergoes rupture when healthy nonischaemic myocardium works efficiently against the weakened necrotic wall. In support of this, cardiac rupture and previous scars were inversely related in our series (table 3 ).

Finally, our series is unusual because we only included patients with acute myocardial infarction who had not been treated with thrombolysis or interventional procedures and who had not undergone previous revascularisation. Our series did not include all fatal cases of acute myocardial infarction seen during the study period, as the cohort was further subselected on the basis of a single pathologist's experience. This population may not therefore be representative of current treatment for acute myocardial infarction. However, the information derived from our study contributes to a greater understanding of thrombogenic substrates in coronary arteries and confirms published data in sudden ischaemic death. ${ }^{10}{ }^{14}$ Furthermore, knowing whether the underlying lesion of thrombosis is a plaque rupture or erosion is not likely to influence the therapeutic approach. To date, it is not known whether an open atheromatous necrotic core after thrombolysis is more thrombogenic than an eroded plaque which remains unmodified following treatment. Furthermore, after effective throm- bolysis, a ruptured plaque may lose the atheromatous necrotic material, thus contributing to a greater likelihood of final lumen patency. In contrast, an eroded plaque is likely to maintain its original size even after thrombolysis. It is most likely that percutaneous transluminal coronary angioplasty will result in similar outcomes in both lesions. This discussion will remain speculative until angiographic studies performed before and after successful thrombolysis begin to assign specific angiographic appearances to the erosion pattern.

CONCLUSION

Plaque erosion is an important local substrate for acute coronary thrombosis in patients with acute myocardial infarction, especially women. Our data suggest that there are differences in plaque formation or local thrombogenic potential in men and women with coronary artery disease.

These data were presented at the American College of Cardiology Meeting, Atlanta, 1998. The study was supported by Grants "Ricerche Finalizzate e Correnti" IRCCS Policlinico San Matteo 1987-96, Pavia, Italy

1 Falk E. Plaque rupture with severe preexisting stenosis precipitating coronary thrombosis. Characteristics of coronary Br Heart f 1983;50:127-34.

2 Davies MJ, Woolf N, Robertson WB. Pathology of acute myocardial infarction with particular reference to occlusive myocardial infarction with particular reference to

3 Arbustini E, Grasso M, Diegoli M, et al. Coronary Arbustini E, Grasso $M$, Diegoli $M$, et al. Coronary
atherosclerotic plaques with and without thrombus in ischemic heart syndromes. A morphologic, immunohistoischemic heart syndromes. A morphologic, immunohistochemical,

4 Depre C, Wijns W, Robert AM, et al. Pathology of unstable plaque: correlation with the clinical severity of acute coronary syndromes. F Am Coll Cardiol 1997;30:694-702.

5 Lee RT, Schoen FJ, Loree HM, et al. Circumferential stress and matrix metalloproteinase 1 in human coronary atherosclerosis. Implications for plaque rupture. Arterioscler Thromb Vasc Biol 1996;16:1070-3.

6 Henney AM, Wakeley PR, Davies MJ, et al. Localization of stromelysin gene expression in atherosclerotic plaques by in situ hybridization. Proc Natl Acad Sci USA 1991;88. 8154-8.

7 Richardson PD, Davies MJ, Born GV. Influence of plaque configuration and stress distribution on fissuring of coronary atherosclerotic plaques. Lancet 1989;ii:941-4.

8 Annex BH, Denning SM, Channon KM, et al. Differential expression of tissue factor protein in directional atherectomy specimens from patients with stable and unstable coronary syndromes. Circulation 1995;91:619-22.

9 Ambrose JA, Winters SL, Arora RR, et al. Coronary angiographic morphology in myocardial infarction: a link between the pathogenesis of unstable angina and myocardial infarction. F Am Coll Cardiol 1985;6:1233-8.

10 Farb A, Burke AP, Tang AL, et al. Coronary plaque erosion without rupture into a lipid core. A frequent cause of coronary thrombosis in sudden coronary death. Circulation 1996;93:1354-63.

11 Arbustini E, Grasso M, Diegoli M, et al. Coronary thrombosis in non-cardiac death. Coron Artery Dis 1993;4: $751-9$.

12 Kaski JC, Chester MR, Chen L, et al. Rapid angiographic progression of coronary artery disease in patients with angina pectoris. The role of complex stenosis morphology. Circulation 1995;92:2058-65.

13 Chen L, Crook JR, Tousoulis D, et al. Complex stenosis morphology predicts late reocclusion during follow-up fter myocardial infarction in patients with patent infarctrelated coronary arteries. Am Heart F 1998;136:877-83.

14 Burke AP, Farb A, Malcolm GT, et al. Effect of risk factors on the mechanism of acute thrombosis and sudden coronary death in women. Circulation 1998;97:2110-16.

15 Lukac P, Kofler K, Waldhor T, et al. Epidemiology of heart wall rupture in myocardial infarct. $Z$ Kardiol 1996;85:776-

16 Kirkpatrick CJ, Breining H. Myocardial infarction, cardiac rupture, and coronary insufficiency in the industrialised Ruhr valley. An autopsy study. Zentralbl Allg Pathol 1983;127:63-73.

17 Solberg S, Nordrum I, Fausa D, et al. Cardiac ruptures in northern Norway. A retrospective study of 104 cases. Acta Med Scand 1988;224:303-10. 